\title{
Support vector machines coupled with proteomics approaches for detecting biomarkers predicting chemotherapy resistance in small cell lung cancer
}

\author{
MINGYONG HAN ${ }^{1}$, JIANJIAN DAI ${ }^{1}$, YING ZHANG ${ }^{1}$, QI LIN ${ }^{1}$, \\ MAN JIANG $^{1}$, XIAOYA XU ${ }^{1}$, QI LIU ${ }^{1}$ and JIHUI JIA ${ }^{2}$ \\ ${ }^{1}$ Cancer Therapy and Research Center, Shandong Provincial Hospital, Shandong University, Jinan 250021; \\ ${ }^{2}$ Department of Microbiology/Key Laboratory for Experimental Teratology of Chinese Ministry \\ of Education, School of Medicine, Shandong University, Jinan 250012, P.R China
}

Received June 4, 2012; Accepted August 27, 2012

DOI: 10.3892/or.2012.2037

\begin{abstract}
The aim of this study was to identify serum protein fingerprints of small cell lung cancer (SCLC) and potential biomarkers related to chemotherapy resistance of SCLC with surface enhanced laser desorption/ionization time of flight mass spectrometry (SELDI-TOF MS). A total of 60 SCLC patients and 48 age- and sex-matched healthy individuals were enrolled. The chemotherapy regimen was cisplatin plus etoposide. All patients received two cycles of chemotherapy. Serum protein profiles were detected using SELDI-TOF MS and the spectra were analyzed with support vector machines (SVMs). Western blotting was performed to verify the results of SELDI-TOF MS. Three top scored peaks, at $\mathrm{m} / \mathrm{z}$ of 6269,9043 and $13124 \mathrm{Da}$, were finally selected as potential biomarkers for detection of SCLC. The SVM classifier separated the SCLC from the healthy samples in the blind test, with a sensitivity of $92.4 \%$ and a specificity of $92.5 \%$. For the 56 eligible chemotherapy patients, 4 had a complete response (7.14\%), 39 patients had a
\end{abstract}

Correspondence to: Dr Jihui Jia, Department of Microbiology/ Key Laboratory for Experimental Teratology of Chinese Ministry of Education, School of Medicine, Shandong University, 44 Wenhua Xi Road, 250012 Jinan, P.R. China

E-mail: jiajihui@163.com

Dr Qi Liu, Cancer Therapy Center, Shandong Provincial Hospital, Shandong University, 324 Jingwuweiqi Road, 250021 Jinan, P.R. China

E-mail: liuqi_liuqi2007@163.com

Abbreviations: $\mathrm{CR}$, complete response; $\mathrm{CV}$, coefficient of variance; $\mathrm{m} / \mathrm{z}$, mass/charge; ROC, receive option curve; SELDI-TOF MS, surface-enhanced laser desorption/ionization time-of-flight mass spectrometry; SVM, support vector machine; UDWT, undecimated discrete wavelet transform

Key words: small cell lung cancer, chemotherapy, proteomics, SELDI-TOF MS partial response $(69.6 \%), 9$ patients had a stable disease $(16.1 \%)$ and 4 patients had a progressive disease (7.14\%). The model constructed using two protein peaks with $\mathrm{m} / \mathrm{z}$ of 8830 and 10468 Da separated the chemotherapy-resistant group from the chemotherapy-sensitive group with a sensitivity of $80.0 \%$ and a specificity of $80.0 \%$. Initial protein database searching identified 10468 Da as S100-A9 which was confirmed by western blotting. The present results suggest that the combination of SELDI-TOF MS with SVM may provide a useful means in the search for serum biomarkers for predicting chemotherapy resistance in patients with SCLC.

\section{Introduction}

Lung cancer is the leading cause of cancer death in the world. In 2011, an estimated 221,000 new cases of lung and bronchial cancer will be diagnosed, and 156,900 deaths are estimated to occur due to the disease. Only approximaely $15.6 \%$ of all lung cancer patients are alive 5 years or more after diagnosis (1). Human lung cancers comprise two major groups, small cell lung cancer (SCLC) and non-small cell lung cancer (NSCLC). SCLC accounts for appoximately $15 \%$ of all lung cancers. When compared with NSCLC, SCLC generally has a more rapid doubling time, a higher growth fraction, and earlier development of widespread metastases (2). Most patients with SCLC present with hematogenous metastases, while only about one third of patients with limited disease confined to the chest (3).

For all patients with SCLC, chemotherapy is an essential component of appropriate treatment. Adjuvant chemotherapy is recommended for those who have undergone surgical resection. For patients with limited stage SCLC and good performance status (PS) (0-2), recommended treatment consists of chemotherapy with concurrent thoracic radiotherapy. For patients with extensive stage disease, chemotherapy alone is the recommended treatment and combination chemotherapy has been shown to be active in SCLC (4). Etoposide and cisplatin (EP) combination is the most commonly used initial chemotherapy regimen. Although progress has been seen with combination chemotherapy, patients with this cancer continue to have a poor prognosis, especially those with disseminated disease (5). 
Since the 80 s, researchers have paid attention to the factors that could be useful in predicting response to treatment and survival. Several studies have attempted to identify clinical, laboratory and molecular markers that may help clinicians and researchers distinguish subgroups of chemotherapy resistant SCLC patients. However, relatively few prognostic factors have been widely accepted as useful predictive markers for individual patients undergoing chemotherapy (6).

Advances in the proteomics study have introduced novel techniques for the screening of new cancer biomarkers and are taking our technology for early diagnosis of cancer diseases to a new horizon (7). Surface-enhanced laser desorption/ionization time-of-flight mass spectrometry (SELDI-TOF MS) is a relatively new approach for analysis of complex biological specimens, including serum $(8,9)$, urine $(10)$, and tears (11). It has proven to be a sensitive system for simultaneously investigating thousands of proteins and identifying the proteomic patterns associated with biological characteristics (12-14).

In this study, we used SELDI-TOF MS to evaluate serum samples from SCLC patients prior to chemotherapy and on day 4 of treatment. The goal was to identify potential serum biomarkers that influence resistance to chemotherapy, and to build a model that could be used to predict chemotherapy resistance, among patients with advanced SCLC treated with standard first-line chemotherapy.

\section{Materials and methods}

Patients and chemotherapy regimen. A total of 60 patients with SCLC and 48 healthy individuals were enrolled between October 2009 and June 2011. All patients had PS of 0-2 and none had brain metastasis. The chemotherapy regimen was etoposide (100 mg/m $\mathrm{m}^{2}$ on days $\left.1-3\right)$, combined with cisplatin ( $75 \mathrm{mg} / \mathrm{m}^{2}$ on day 1), 3-week cycle. All patients received two cycles of chemotherapy. Patients who had received prior chemotherapy and radiotherapy were ineligible for the study. The criteria for eligibility included confirmed disease (measurable or non-measurable), $\geq 18$ years of age, adequate hematological function as indicated by a white cell count of $\geq 4000 / \mathrm{cm}^{3}$ and a platelet count of $\geq 100,000 / \mathrm{cm}^{3}$, and normal hepatic function as indicated by a bilirubin concentration that did not exceed $17 \mathrm{mmol} / \mathrm{l}$. Tumor response was evaluated after two courses by repeating appropriate radiographic studies on measurable lesions determined before inclusion. Treatment response was evaluated according to the World Health Organization criteria (15). Briefly, complete response was defined as the absence of disease at all known sites for at least 4 weeks. Partial response was defined as a $50 \%$ reduction in the sum of the perpendicular diameters of all measurable lesions, lasting $\geq 4$ weeks. Progressive disease was defined as either a $25 \%$ increase in the area of any one lesion over the prior measurement, or the development of one or more new lesions. Others were defined as stable disease. Chemotherapy resistance includes stable disease and progressive disease. The objective response in the malignant pleural effusion was evaluated by CT scan after two courses using the response criteria of the Japan Lung Cancer Society (16). Complete response was defined as the complete disappearance of pleural fluid and negative cytological findings for 4 weeks. Partial response was defined as a distinguishable decrease or no increase in pleural fluid and negative cytological findings for
4 weeks. No response was defined as failure to meet the above criteria. Patients with SCLC had an average age of 53.6 years (range 44-73 years; 39 men and 17 women) and consisted of 38 and 18 patients suffering from limited and extensive disease. Staging of SCLC was carried out according to the Veteran's Administration lung cancer group staging system (17), limited stage was defined as a disease as a confined to one hemithorax including mediastinal lymph nodes and/or supraclavicular lymph nodes. Extensive disease was defined by opposition to the criteria of limited disease. The study was approved by the Institutional Ethics Committee and conducted in accordance with Helsinki declaration. Patients gave informed consent.

Serum sample. Blood samples were collected by venipuncture at the Shandong Provincial Hospital of Shandong University. Two milliliters of whole blood were collected from SCLC patients before chemotherapy and on day 4 of chemotherapy during the first course of treatment. The blood was stored at $4^{\circ} \mathrm{C}$ within $1 \mathrm{~h}$ following collection and later centrifugated for $20 \mathrm{~min}$ at $4000 \mathrm{~g}$. Serum samples were divided into $100 \mathrm{ml}$ aliquots and stored at $-80^{\circ} \mathrm{C}$ until use.

Proteinchip array analysis. All serum specimen tubes were thawed in wet ice and centrifugated at $5000 \mathrm{rpm}$ for $5 \mathrm{~min}$, sampled for $10 \mu \mathrm{l}$ and buffered with $90 \mu \mathrm{l}$ of $0.5 \%$ CHAPS (pH 7.4) for $5 \mathrm{~min}$, to which was added $100 \mu \mathrm{l}$ Cibcron blue 3.0 G (Sigma, St. Louis, MO, USA) and vortexed at $4^{\circ} \mathrm{C}$ for $60 \mathrm{~min}$ on a platform shaker, then $50 \mu 1$ samples were taken and diluted with $20 \mathrm{mmol} / \mathrm{l} \mathrm{HEPES}$ to $240 \mu \mathrm{l}$ (total reaction volume) and applied to each spot on the Protein Chip Array by a 96-well bioprocessor (Ciphergen, which can hold 12 pieces of chips). After the samples were allowed to bind at $4^{\circ} \mathrm{C}$ for $60 \mathrm{~min}$ on a platform shaker, the array was washed twice with $200 \mu \mathrm{l}$ of $20 \mathrm{mmol} / \mathrm{l} \mathrm{HEPES}$ for $5 \mathrm{~min}$, followed by two quick rinses with $200 \mu \mathrm{l}$ of distilled $\mathrm{H}_{2} \mathrm{O}$. After air-drying, $0.5 \mu \mathrm{l}$ of $\mathrm{CHCA}$ (saturation in $50 \%$ acetonitrile and $0.5 \%$ trifluoroacetic acid) were applied twice to each spot. Proteins bound to the H4 chips (through hydrophobic amino acids) were detected with the ProteinChip Reader. Data were collected by averaging 80 laser shots with intensity of 155 and detector sensitivity of 8 .

Bioinformatics analysis. Our method analyzing all the data relies on the undecimated discrete wavelet transform (UDWT) as a first step to denoise of spectra. The UDWT method is based on the version 2.4 of the Rice Wavelet Toolbox (RWT). Wavelets have been used previously to denoise signals in a number of contexts, including magnetic resonance imaging, ultrasound blood flow. It has been reported to yield better visual and qualitative denoising. After denoising, the spectra performed baseline correction (by fitting a monotone local minimum curve) and mass calibration (adjusting the intensity scale according to 3 labeled peaks that appears in all the selected spectra). The proteomic peaks detected and quantified by an algorithm locates all local maxima height in each denoised, baseline-corrected, calibrated spectrum. Then the peaks are filtered by the signalto-noise ratio $>3$ (the signal-to-noise ratio of a peak is estimated as the height above baseline divided by a wavelet-defined noise). To match peaks across spectra, we pooled the list of detected peaks and combined peaks in relative mass by $0.3 \%$, and the percentage of each peak appears in spectra is specified to 10 . 
Table I. The descriptive statistics of 3 protein peaks in pattern distinguishing SCLC patients from HP.

\begin{tabular}{rcccccc}
\hline $\mathrm{m} / \mathrm{z}$ & AUC & P-value $\left(\mathrm{x} 10^{-5}\right)$ & HP & SCLC & Mean S/N of healthy & Mean S/N of cancer \\
\hline 6269 & 0.86 & 0.02 & $11.77 \pm 4.98$ & $4.09 \pm 2.24$ & 12.03 & 3.88 \\
9043 & 0.84 & 0.08 & $3.67 \pm 4.21$ & $12.68 \pm 7.12$ & 2.96 & 14.89 \\
13124 & 0.77 & 0.11 & $2.95 \pm 2.33$ & $7.87 \pm 3.29$ & 2.64 & 6.12 \\
\hline
\end{tabular}

S/N, signal/noise; HP, healthy people; SCLC, small cell lung cancer.

The matched peak across spectra is defined as peaks cluster. The spectra that do not have a peak within a given cluster will be assigned a maximal height in the cluster for the peak.

SVM classifier. SVM is a new machine learning approach originally proposed and developed by Vapnik (18). SVM applications are being actively pursued in various areas recently, from face recognition to genomics (19). It is a powerful tool for analyzing complex data derived from SELDI-TOF MS. We constructed a non-linear SVM classifier with a radial based function (RBF) kernel, and with the parameter Gamma 0.6, being the cost of the constrain violation 19 to discriminate the different groups. Cross-validation approach (10-fold) was applied to estimate the accuracy of the classifier. This approach randomly selected the $9 / 10$ of all the samples to be the blinded training set, and the remaining 1/10 samples to be the test set and repeated the procedure 10 times. SVM classifier is based on the shareware program OSU_SVM v.3.00 Toolbox of Junshui Ma and Yi Zhao.

Feature selection and model establishment. The power of each peak in discriminating different groups was estimated by receive option curve (ROC). The greater area under the curve value of the peak shows the higher relative importance value of the ability to accurately distinguish the different groups. The peaks with lower area under the curve values are excluded. To further select the set of candidate biomarkers, a stepwise approach was used for training many SVM. The top 1 peak that had the highest ability to predict the two groups (having the highest area under curve values) was selected as single input to build the SVM. The discriminating ability of this SVM was estimated by the accuracy of blind test set. Then, the top 2 peaks were inputted to the SVM and the accuracy was calculated. The following peaks were added in input stepwise fashion to train the SVM and the accuracy was calculated. In this way, many models with different peaks were built. The peaks inputted to the model with highest accuracy were selected as the set of potential biomarkers. And the SVM with the highest accuracy was selected for detecting SCLC.

Western blot analysis. Briefly, serum proteins were separated by electrophoresis in a SDS-polyacrylamide gel (SDS-PAGE), and subsequently transferred onto polyvinylidene fluoride membranes. The membranes were blocked for $1 \mathrm{~h}$ at room temperature in $5 \%$ skim milk, then incubated for overnight at $4^{\circ} \mathrm{C}$ with mouse anti-human S100-A9 antibody (Novus Biologicals, USA). After washing with PBS, the membranes were incubated with rabbit anti-mouse antibody for $1 \mathrm{~h}$ at $37^{\circ} \mathrm{C}$. Finally, the membranes were developed using a Super Signal
West PicoChemiluminescent Substrate kit (Pierce, USA) followed by Imaging System.

\section{Results}

Reproducibility of the experiment. The reproducibility of the SELDI spectra, i.e., mass and intensity intraassay and interassay, was determined with the pooled normal serum quality control (QC) sample. A total of four proteins in the range of 2-30 kDa observed on spectra randomly selected over the course of the study were used to calculate the mean coefficient of variance (CV). The intra- and interassay mean CV for mass were 0.3 and $0.5 \%$, respectively, and the intra- and interassay mean $\mathrm{CV}$ for the normalized intensity were 10 and $14 \%$, respectively. There was little variation with day-to-day sampling and instrumentation or chip variations.

Serum SELDI profiles of lung cancer vs. healthy controls. After noise filtering and peak cluster identification, 108 mass peaks were detected in the training set. These qualified peaks detected from the SCLC and healthy control groups were ranked by ROC. The top 10 peaks with higher area under curve values were selected, randomly combined, and fed into SVM. The accuracy of each combination in distinguishing SCLC from healthy control was analyzed, and the SVM model with the highest accuracy was used as the diagnostic model. This model, comprised three potential biomarkers with mass/charge $(\mathrm{m} / \mathrm{z})$ of 6269, 9043 and $13124 \mathrm{Da}$, respectively. The peaks with $\mathrm{m} / \mathrm{z}$ of 9043 and 13124 Da were highly expressed in SCLC but weakly expressed in healthy people; the peaks with $\mathrm{m} / \mathrm{z}$ of $6269 \mathrm{Da}$ appeared to be expressed in a contrasting way, as shown in Fig. 1. The descriptive statistics of these three peaks are shown in Table I.

The diagnostic model was trained with 72 samples and tested with the remained 36 samples. Through the 10 -fold cross-validation SVM, the specificity is $96.4 \%$, the sensitivity is $93.5 \%$ in the training set. By analyzing the blind serum samples, it yielded a sensitivity of $92.4 \%$ and a specificity of $92.5 \%$ (Table II).

Chemotherapy resistance and chemotherapy response. Among the 60 patients enrolled in the study, 4 patients were found ineligible. Four patients underwent reduction of the etoposide dose because of grade 4 neutropenia lasting for 3 days. For the 56 eligible patients, 4 had a complete response $(7.14 \%)$, 39 patients had a partial response (69.6\%); 9 patients had a stable disease $(16.1 \%)$ and 4 patients had a progressive disease $(7.14 \%)$. There were 13 patients with resistance to chemotherapy and 43 patients with chemotherapy sensitivity. 

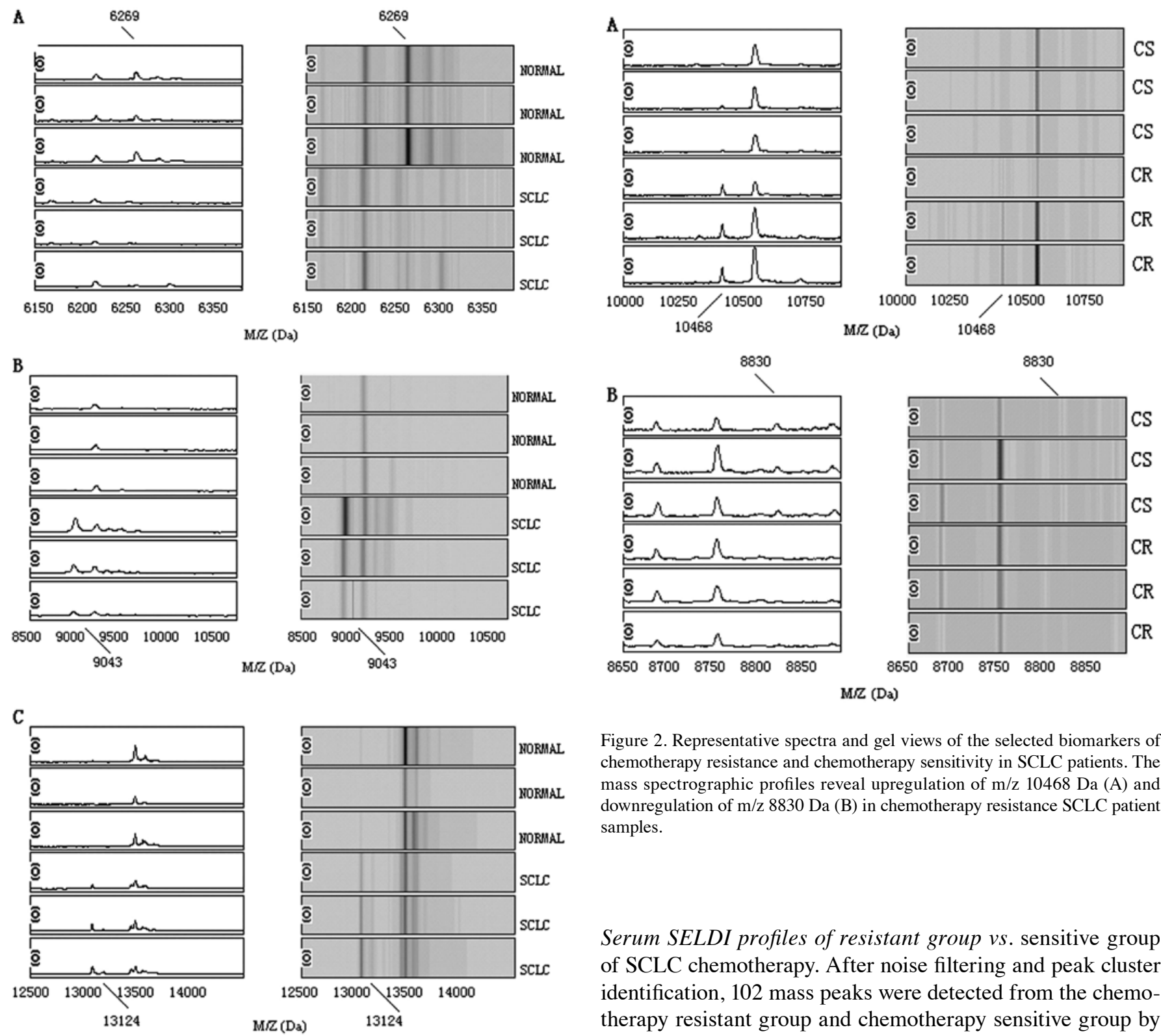

Figure 2. Representative spectra and gel views of the selected biomarkers of chemotherapy resistance and chemotherapy sensitivity in SCLC patients. The mass spectrographic profiles reveal upregulation of m/z $10468 \mathrm{Da}$ (A) and downregulation of $\mathrm{m} / \mathrm{z} 8830 \mathrm{Da}(\mathrm{B})$ in chemotherapy resistance SCLC patient samples.

Figure 1. Representative spectra and gel views of the selected biomarkers of lung cancers patients and healthy individuals. The mass spectrographic profiles reveal downregulation of $\mathrm{m} / \mathrm{z} 6269 \mathrm{Da}(\mathrm{A})$ and upregulation of $\mathrm{m} / \mathrm{z}$ 9043 Da (B), m/z 13124 Da (C) in SCLC patients.

Table II. The predicted results of 10-fold cross-validation SVM for distinguishing SCLC patients from HP.

\begin{tabular}{lcclcc}
\hline & \multicolumn{2}{c}{ Training set $(72 \times 10)$} & & \multicolumn{2}{c}{ Test set (36x10) } \\
\cline { 2 - 3 } \cline { 5 - 6 } & SCLC & HP & & SCLC & HP \\
\hline SCLC (60x10) & 388 & 27 & & 171 & 14 \\
HP (48x 10) & 11 & 294 & & 13 & 162 \\
Sensitivity (\%) & $93.5[388 /(388+27)]$ & & $92.4[171 /(171+14)]$ \\
Specificity (\%) & $96.4[294 /(294+11)]$ & & $92.5[162 /(162+13)]$ \\
Positive value & $97.2[388 /(388+11)]$ & & $92.9[171 /(171+13)]$ \\
$(\%)$ & & & &
\end{tabular}

SCLC, small cell lung cancer. HP, healthy people.

Serum SELDI profiles of resistant group vs. sensitive group of SCLC chemotherapy. After noise filtering and peak cluster identification, 102 mass peaks were detected from the chemotherapy resistant group and chemotherapy sensitive group by ROC. The top 10 peaks with higher area under curve values were selected, randomly combined, and fed into the SVM. The accuracy of each combination for distinguishing the chemotherapy resistant group and chemotherapy sensitive group was analyzed. The SVM model with the highest accuracy was used as the diagnostic model. This model comprised two potential biomarkers with a m/z of 8830 and $10468 \mathrm{Da}$. The peaks with $\mathrm{m} / \mathrm{z}$ of $10468 \mathrm{Da}$ were highly expressed in the chemotherapy resistant group but weakly expressed in the chemotherapy sensitive group; the peaks with $\mathrm{m} / \mathrm{z}$ of $8830 \mathrm{Da}$ appeared to be expressed in opposite ways (Fig. 2).

The construction and validation of chemotherapy resistance predictive model. The predictive model was trained using 36 samples and tested with the remaining 20 samples. Following the 10-fold cross-validation SVM, the specificity was $75.0 \%$, and the sensitivity was $85.7 \%$ in the training set. In the blind test sets, 12 out of 15 chemotherapy sensitive group samples and 4 of 5 chemotherapy resistant group were correctly classified by analyzing the blind serum samples. This yielded a sensitivity of $80.0 \%$, and a specificity of $80.0 \%$ (Table III). 
Table III. The predicted results of patterns distinguishing chemotherapy resistance from chemotherapy sensitivity.

\begin{tabular}{|c|c|c|c|c|}
\hline & \multicolumn{2}{|c|}{ Training set (36) } & \multicolumn{2}{|c|}{ Testing set (20) } \\
\hline & $\mathrm{CR}$ & $\mathrm{CS}$ & $\mathrm{CR}$ & $\mathrm{CS}$ \\
\hline CR (13) & 24 & 4 & 123 & \\
\hline CS (43) & 2 & 6 & 1 & 4 \\
\hline Sensitivity (\%) & \multicolumn{2}{|c|}{$85.7[24 /(24+4)]$} & \multicolumn{2}{|c|}{$80.0[12 /(12+3)]$} \\
\hline Specificity (\%) & \multicolumn{2}{|c|}{$75.0[6 /(6+2)]$} & \multicolumn{2}{|c|}{$80.0[4 /(4+1)]$} \\
\hline
\end{tabular}

$\mathrm{CR}$, chemotherapy resistance; CS, chemotherapy sensitivity.

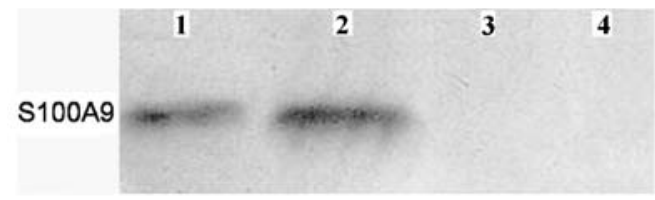

Figure 3. Validation of identified proteins by western blotting. The analysis of two chemotherapy resistance and two chemotherapy sensitivity samples $(20 \mu \mathrm{l}$ each). Samples were selected based on the SELDI analysis for the presence of a peak cluster at m/z 10468 (S100-A9) (1,2 chemotherapy resistance samples) or absence of this $\mathrm{m} / \mathrm{z}$ peaks ( 3,4 chemotherapy sensitivity samples). Molecular weight indicators are shown on the left, and the target proteins on the right of the blots.

Identification of S100A9. According to molecular weight and electric charge of different protein peaks, the peaks identified were retrieved in SWISS-PROT database (http://www. uniprot.org/) (20) for their identification. The protein peak $\mathrm{m} / \mathrm{z}$ $10468 \mathrm{Da}$ was identified as protein S100-A9. To confirm this, we performed western blot analysis and found that S100-A9 was indeed at higher level in chemotherapy resistant group (Fig. 3).

\section{Discussion}

In this study, we used the integrated approach of SELDI-TOF MS and SVM tools to analyze the large data of spectra. We established a protein fingerprint pattern for SCLC with three potential biomarkers, $\mathrm{m} / \mathrm{z}$ at 6269, 9043 and $13124 \mathrm{Da}$ to distinguish SCLC from healthy controls. The specificity and the sensitivity of this pattern were 92.5 and $92.4 \%$, respectively. This result showed that the selection of a combination of multiple proteins obtained from SELDI may become a potential diagnostic approach. The peaks with $\mathrm{m} / \mathrm{z}$ of 9043 and 13124 Da were highly expressed in lung cancer but weakly expressed in healthy people, so the two protein peaks remain of interest to be further investigated.

The commonly used biomarkers for clinical diagnosis and prognosis in patients with lung cancer today are carcinoembryonic antigen (CEA), cytokeratin-19 fragments (CYFRA-211), and NSE (21). However, all these biomarkers have a poor positive predictive value especially during the early-stage of lung cancer, and some biomarkers are not specific to lung cancer. In light of the multifactorial nature of cancer, it is very likely that a combination of several markers will be necessary to improve the detection and diagnosis of lung cancer. SELDI-TOF MS ProteinChip technology is a new technique that allows multiple serum samples obtained directly from patients to be analyzed in a relatively short time (22). It is a high-throughput approach used to generate protein expression profiles, which in combination with bioinformatics tools to extract information for biomarker discovery, has been essential in identifying novel protein biomarkers.

As we hypothesized, changes in serum proteins will occur after chemotherapy and such changes could be detected with SELDI-TOF MS. In this study, we used the integrated approach of SELDI-TOF MS and SVM tools to compare protein spectra of chemotherapy resistant patients with chemotherapy sensitive patients. We established a protein fingerprint patterns for predictive chemotherapy resistant in SCLC patients. Two potential biomarkers, $\mathrm{m} / \mathrm{z}$ at 10468 and $8830 \mathrm{Da}$, were identified and constructed a pattern to distinguish chemotherapy resistant patients from chemotherapy sensitive patients. The specificity and the sensitivity of this pattern were 80.0 and $80.0 \%$, respectively. This result showed that selection of a combination of multiple proteins identified by from SELDI may become a potential predictive approach.

Using SELDI-TOF MS profiling, we examined proteomic changes in the serum of patients with SCLC before and after chemotherapy. The peaks with $\mathrm{m} / \mathrm{z}$ of $10468 \mathrm{Da}$ were highly expressed in the chemotherapy resistant group, but weakly expressed in the chemotherapy sensitive group. Thus, the protein peak may be chemotherapy resistant protein and of interest for further investigation. The peaks with $\mathrm{m} / \mathrm{z}$ of $8830 \mathrm{Da}$ were highly expressed in the chemotherapy sensitive group, but weakly expressed in the chemotherapy resistant group. The presence of the protein peak suggests that the protein may represent a cytotoxic therapy induced response that may originate from the carcinoma itself or be a host response to cytotoxic therapy.

The protein peak m/z $10468 \mathrm{Da}$ was identified as protein S100-A9, and further confirmed by western blot analysis, S100 protein family consists of at least 24 members, and are small $\mathrm{Ca} 21$ binding proteins that participate in many cellular functions including tumor growth (23). Protein S100-A9 (S100A9), was suggested to be a marker for pancreatic cancer, inflammatory bowel disease, lung adenocarcinoma and breast cancer (24). Our results suggested that the protein S100-A9 may be not only a tumor marker but also for the chemotherapy resistance in SCLC.

One of the challenges in the analysis of SELDI-TOF MS generated data is to reduce the false protein peaks, in which the discriminatory power is due to random variation (25). The SVM classification technique used in the study is a sophisticated machine learning method based on the statistical theory. The SVM can solve problems such as the generalization of the medium and small samples in pattern recognition, pattern selection, and over-fitting (26-30).

In this study, we instituted various preventive measures to avoid generation of biased results caused by artifacts related to the nature of the clinical samples. All serum samples were collected and processed within the same clinical and laboratory settings. To avoid variation in the procedure, freshly collected sera were immediately aliquoted, stored at $-80^{\circ} \mathrm{C}$, and thawed only once. Standard protocols must be developed to minimize unwanted fluctuation, and CVs between proteinchips must be 
calculated by using common peaks across different spectra. The use of paired serum samples from individual patients in the study removed most of genetic and environmental variables and made it likely that the changes in protein profile reflected the disease state more exactly. We also used quality control serum to allow detection of any unusual features during the process. Such precautions led to very good reproducibility of the protein peak patterns.

In conclusion, we have shown that using proteomics approaches in combination with bioinformatics tools could distinguish small cell lung cancer patients from healthy controls with relatively high sensitivity and specificity. The combination of SELDI-TOF MS with SVM could identify new potential tumor markers for chemotherapy resistant. Further research is needed to elucidate the sequence of the interesting peptides identified in our current study, and to confirm our current findings in larger cohorts of study samples.

\section{Acknowledgements}

This study was supported by General Programs of Natural Science Foundation of Shandong Province (No.BS2009SW050) and Key Development Program for Basic Research of Shandong Province (2007GG20002007).

\section{References}

1. Siegel R, Ward E, Brawley O and Jemal A: Cancer statistics, 2011: the impact of eliminating socioeconomic and racial disparities on premature cancer deaths. CA Cancer J Clin 61: 212-236, 2011.

2. Gaspar LE, McNamara EJ, Gay EG, Putnam JB, Crawford J, Herbst RS and Bonner JA: Small cell lung cancer: Prognostic factors and changing treatment over 15 years. Clin Lung Cancer 13: 115-122, 2011.

3. Neal JW, Gubens MA and Wakelee HA: Current management of small cell lung cancer. Clin Chest Med 32: 853-863, 2011.

4. Guo H, Deng Q, Wu C, et al: Variations in HSPA1B at $6 \mathrm{p} 21.3$ are associated with lung cancer risk and prognosis in Chinese populations. Cancer Res 71: 7576-7581, 2011.

5. Szkorupa M, Klein J, Bohanes T, Neoral C, Kolek V and Grygárková I: Neoadjuvant chemotherapy and surgical treatment in advanced stages of non-small cell lung cancer. Rozhl Chiv 90: 433-439, 2011.

6. Zeng HZ, Qu YQ, Liang AB, et al: Expression of CD147 in advanced non-small cell lung cancer correlated with cisplatinbased chemotherapy resistance. Neoplasma 58: 449-454, 2011.

7. Titulaer MK, Siccama I, Dekker LJ, van Rijswijk AL, Heeren RM, Sillevis Smitt PA and Luider TM: A database application for preprocessing, storage and comparison of mass spectra derived from patients and controls. BMC Bioinformatics 7: 403, 2006.

8. Jemal A, Murray T, Samuels A, Ghafoor A, Ward E and Thun MJ: Cancer statistics. 2003. CA Cancer J Clin 53: 5-26, 2003.

9. Engwegen JY, Gast MC, Schellens JH and Beijnen JH: Clinical proteomics: searching for better tumour markers with SELDI-TOF mass spectrometry. Trends Pharmacol Sci 27: 251-259, 2006.

10. Mosley K, Tam FW, Edwards RJ, Crozier J, Pusey CD and Lightstone L: Urinary proteomic profiles distinguish between active and inactive lupus nephritis. Rheumatology 45: 1497-1504, 2006.
11. Tomosugi N, Kitagama K, Takahashi N, Sugai S and Ishikawa I: Diagnostic potential of tear proteome patterns in Sjögren's syndrome. J Proteome Res 4: 820-825, 2005.

12. Yu Y, Chen S, Wang LS, et al: Prediction of pancreatic cancer by serum biomarkers using surface-enhanced laser desorption/ ionization-based decision tree classification. Oncology 68: 79-86, 2005.

13. Koopmann J, Zhang Z, White N, et al: Serum diagnosis of pancreatic adenocarcinoma using surface-enhanced laser desorption and ionization mass spectrometry. Clin Cancer Res 10: 860-868, 2004.

14. Merchant $M$ and Weinberger SR: Recent advancements in surface enhanced Iaser desorption ionization-time of fIight-mass spectrometry. Electrophoresis 21: 1164-1177, 2000.

15. World Health Organization: WHO handbook for reporting results of cancer treatment. World Health Organization, Geneva, WHO Offset Publication No. 48, 1979.

16. Japan Lung Cancer Society: Classification of lung cancer. 1st edition. Kanehara \& Co., Ltd., Tokyo, pp49-52, 2000.

17. Li J, Zhang Z, Rosenzweig J, Wang YY and Chan DW: Proteomics and bioinformatics approaches for identification of serum biomarkers to detect breast cancer. Clin Chem 48: 1296-1304, 2002.

18. Liu Y: Active learning with support vector machine applied to gene expression data for cancer classification. J Chem Inf Comput Sci 44: 1936-1941, 2004.

19. Lin HH, Han LY, Cai CZ, Ji ZL and Chen YZ: Prediction of transporter family from protein sequence by support vector machine approach. Proteins 62: 218-231, 2006.

20. UniProt Consortium: The universial protein resource (Uniprot) in 2010: Nucleic Acids Res 38: D142-D148, 2010.

21. Lee JH and Chang JH: Diagnostic utility of serum and pleural fluid carcinoembryonic antigen, neuron specific enolase and cytokeratin 19 fragments in patients with effusion from primary lung cancer. Chest 128: 2298-2303, 2005.

22. Seibert V, Wiesner A, Buschmann T and Meuer J: Surfaceenhanced 1 aserdesorption ionization time-of-flight mass spectrometry (SELDI TOF-MS) and ProteinChip technology in proteomics research. Pathol Res Pract 200: 83-94, 2004.

23. Kawai H, Minamiya Y and Takahashi N: Prognostic impact of S100A9 overexpression in non-small cell lung cancer. Tumor Biol 32: 641-646, 2011.

24. Yang WS, Moon HG, Kim HS, Choi EJ, Yu MH, Noh DY and Lee C: Proteomic approach reveals FKBP4 and S100A9 as potential prediction markers of therapeutic response to neoadjuvant chemotherapy in patients with breast cancer. J Proteome Res 11: 1078-1088, 2012.

25. Petricoin EF and Liotta LA: SELDI-TOF-based serum proteomic pattern diagnostics for early detection of cancer. Curr Opin Biotechnol 5: 24-30, 2004.

26. Somorjai RL, Dolenko B and Baumgartner R: Class prediction and discovery using gene microarray and proteomics mass spectroscopy data: curses, caveats, cautions. Bioinformatics 19: 1481-1491, 2003.

27. Furey TS, Cristianini N, Duffy N, Bednarski DW, Schummer M and Haussler D: Support vector machine classification and validation of cancer tissue samples using microarray expression data. Bioinformatics 16: 906-914, 2000.

28. Byvatov E and Schneider G: Support vector machine applications in bioinformatics. Appl Bioinformatics 2: 67-77, 2003.

29. Jorissen RN and Gilson MK: Virtual screening of molecular databases using a support vector machine. J Chem Inf Model 45: 549-561, 2005.

30. Wiesner A: Detection of tumor markers with proteinChip technology. Curr Pharm Biotechnol 15: 45-67, 2004. 